\title{
Comparative Study between Intrathecal Dexmedetomidine and Intrathecal Magnesium Sulfate for the Prevention of Post-Spinal Anesthesia Shivering in Lower Limb Orthopedic Surgeries: A Randomized Controlled Trial
}

\author{
GIHAN SEIF-ELNASR MOHAMED, M.D.; FADY ADIB ABD ELMALEK, M.D.; FAROUK KAMAL, M.D. and \\ ASMAA M. SAAD ABD EL-HAFIZ, M.Sc.
}

The Department of Anesthesia, Intensive Care and Pain Management, Faculty of Medicine, Ain Shams University

\begin{abstract}
Background: Shivering is a common and unpleasant perioperative event following general as well as neuraxial anesthesia. It has been reported in $40-70 \%$ of regionally anesthetized patients. Shivering is a physiological compensatory response to core hypothermia precipitated by anesthetic induced thermoregulatory impairment, exposure to a cool environment, infusion of unwarmed fluids and evaporation from exposed surfaces. However, some shivering like tremors is not thermoregulatory.
\end{abstract}

Aim of Study: To compare the efficacy of intrathecal dexmedetomidine versus intrathecal magnesium sulphate when added to hyperbaric bupivacaine for reducing the severity and incidence of post-spinal shivering in lower limb orthopedic surgeries.

Patients and Methods: We compared the efficacy of intrathecal dexmedetomidine versus intrathecal magnesium sulphate for reducing the severity and incidence of post-spinal shivering in lower limb orthopedic surgeries. This study was a randomized controlled trial, 105 adult patients (ASA I-II) of both sexes, aged 18-45 years, were scheduled for lower limb orthopedic surgeries in Ain Shams University Hospitals. Patients were randomly allocated into three equal groups; each group consists of 35 patients and had spinal anesthesia with one of the following mixture:

- In group A, spinal anesthesia was performed with $3.5 \mathrm{ml}$ of $0.5 \%$ hyperbaric bupivacaine (17.5mg), plus $5 \mu \mathrm{g}$ dexmedetomidine in $0.5 \mathrm{ml}$ normal saline.

- In group B, 3.5ml of $0.5 \%$ hyperbaric bupivacaine (17.5mg), plus $25 \mathrm{mg} \mathrm{MgSO} 4$ in $0.5 \mathrm{ml}$ normal saline was injected intrathecally.

- In group C, $3.5 \mathrm{ml}$ of $0.5 \%$ hyperbaric bupivacaine (17.5mg), plus $0.5 \mathrm{ml}$ of normal saline was injected intrathecally.

Results: Our results showed that all patients in the three groups were comparable regarding demographic data (including age, sex, weight and height) and intraoperative hemody-

Correspondence to: Dr. Asmaa M. Saad Abd El-Hafiz, E-Mail: asmaa.m.s.1991@gmail.com namics (SBP, DBP and HR). Regarding temperature, though difference in decreased core temperature was clinically insignificant, there was statistical significant difference between the three groups at almost all times as temperature at dexmedetomidine group was higher than magnesium group but the temperature at controlled group was lower than other two groups. Regarding shivering, we found that $\mathrm{C}$ group showed statistically significant higher number of total patients who developed shivering than B group and A group. 9 patients $(25.7 \%)$ in dexmedetomidine group (group A), 12 patients $(34.3 \%$ ) in magnesium sulfate group (group B), and 20 patients $(57.1 \%)$ in control group (group C).

Conclusion: Our study viewed that intrathecal administration of dexmedetomidine or magnesium sulphate as adjuvant to heavy bupivacaine in spinal anesthesia, reduced incidence and severity of shivering in patients presented for lower limb orthopedic surgery with non-significant difference among both adjuvants.

Key Words: Post-anesthetic care unit-Intrathecal dexmedetomidine - Intrathecal magnesium sulfate.

\section{Introduction}

SPINAL anesthesia is the most common performed technique in prolonged lower limb surgeries. Bupivacaine is routinely used with the addition of number of adjuvants to increase its duration and potency of analgesia such as opioids [1]

Shivering is a common and unpleasant perioperative event following general as well as neuraxial anesthesia. It has been reported in $40-70 \%$ of regionally anesthetized patients. Shivering is a physiological compensatory response to core hypothermia precipitated by anesthetic induced thermoregulatory impairment, exposure to a cool environment, infusion of unwarmed fluids and evaporation from exposed surfaces. However, some shivering like tremors is not thermoregulatory [2] 
Post-spinal shivering presents by different degrees ranging from piloerection in the mild form up to generalized skeletal muscle contraction in the severe form that interferes with pulse rate, blood pressure and electrocardiographic (ECG) monitoring [3].

Shivering increases the metabolic rate, the oxygen consumption and carbon dioxide production with lactic acidosis which is hazardous in patients with poor cardiac and/or respiratory reserves. It also increases intraocular and intracranial pressures and leads to hypertension, tachycardia, patient discomfort, increased wound pain, delayed wound healing, and delayed discharge from the postanesthetic care unit (PACU). That is why its primary prevention and prompt control are warranted [4].

Dexmedetomidine is a highly selective alpha2-adrenoreceptor agonist with potent effects on the central nervous system, decreasing the sympathetic tone [5]. It has been effectively used intravenously for the treatment and prevention of shivering following SA without any major adverse effects in several studies [6]. Few trials have examined intrathecal dexmedetomidine for the prevention of post-SA shivering [7].

On the other hand, magnesium sulphate has been shown to suppress postoperative shivering, it is suggested that it reduces the shivering threshold. The drug not only exerts a central effect, but is also a mild muscle relaxant and may thus simultaneously reduce the gain of shivering (incremental shivering intensity with progressing hypothermia) [8].

\section{Aim of the work:}

The aim of the present trial is to compare the efficacy of intrathecal $5 \mathrm{gg}$ dexmedetomidine versus intrathecal $25 \mathrm{mg}$ magnesium sulphate when added to $3.5 \mathrm{ml}$ of $0.5 \%$ hyperbaric bupivacaine $(17.5 \mathrm{mg}$ ) in $0.5 \mathrm{ml}$ normal saline for reducing the severity and incidence of post-spinal shivering in lower limb orthopedic surgeries.

\section{Patients and Methods}

This is a randomized controlled trial set at Ain Shams University Hospitals over a period of 3-6 months on 105 patients who were scheduled to undergo lower limb orthopedic surgeries receiving spinal anesthesia at Ain Shams University Hospitals during 2020. The patients divided into three groups; group A included patients who received dexmedetomidine as adjuvant to $0.5 \%$ heavy bupivacaine, group B included patients who received magnesium sulphate as adjuvant to $0.5 \%$ heavy bupivacaine and group $\mathrm{C}$ who received $0.5 \%$ heavy bupivacaine as a control group.

\section{Inclusion criteria:}

Our inclusion criteria were: No sex predilection. Age between 18 and 45 years old. Patients with American Society of Anesthesiologists (ASA) physical status classification I and II. Anticipated duration of surgery to be not more than 120 minutes. Normothermia $\left(36.5^{\circ} \mathrm{C}-37.2^{\circ} \mathrm{C}\right)$.

\section{Exclusion criteria:}

Our exclusion criteria were: Refusal of procedure or participation in the study. Tempretaure below $36.5^{\circ} \mathrm{C}$ or above $37.2^{\circ} \mathrm{C}$. Obese patients $\left(\mathrm{BMI}>30 \mathrm{~kg} / \mathrm{m}^{2}\right)$. Expected intraoperative bleeding and blood transfusion. Allergy to dexmedetomidine or magnesium sulphate. Drug or alcohol abuse. Patients who are taking 0 blockers, vasodilators, gonadotropin, tramadol, clonidine or pregabalin before the surgery. Patients with any contraindication to neuraxial block (relative or absolute). Bleeding disorders.

\section{Sampling method:}

Non-probability consecutive sample. Using PASS 11 program for sample size calculation and assuming incidence of shivering in study groups are $79 \%, 31 \%$ and $6 \%$, sample size of 35 patients /group can detect the difference between 3 groups setting power at $80 \%$ and a-error at 0.05 .

\section{Methods:}

A written informed consent was obtained from all participants or their guardians prior to screening and enrolment. Benefits from participation in the research were explained to all patients. The study's protocol gained the ethical approval of the Ethics Committee of Ain Shams University Hospitals.

All cases were assessed for any hemodynamic abnormalities in the preoperative room. All participants underwent full history taking, clinical examination, routine investigations including CBC, liver function tests, renal function tests and coagulation profile.

Randomization was done through opaque and well-sealed envelopes. The sequence generation was done with a computer. The number was printed on envelopes and the group was written on the card together with the serial number. When the patient arrived. The envelope was opened to see the group that would be assigned. A drug solution 
was prepared by a doctor who did not participate in the study, and drugs were filled in pre-coded syringes and given to the anesthetist. Patients were also blinded for the administered drug.

After entering the operating room, an IV cannula (18 Gauge) was inserted under complete aseptic conditions. Standard ASA monitoring [electrocardiography (ECG), non-invasive arterial blood pressure (NABP), pulse rate, and pulse oximetry monitoring] in addition to temperature monitoring was applied.

Before performing SA, all the patients were infused with $500 \mathrm{~mL}$ of Ringer lactate, which had been warmed to $37^{\circ} \mathrm{C}$. For all participants, SA was conducted by an expert anesthesiologist who was unaware of the patients' grouping and was not involved in data collection.

\section{Conduct of anesthesia:}

Under all aseptic precautions, a midline spinal puncture was performed at the L3-L4 or L4-L5 level in a sitting position using a 25 gauge Quincke spinal needle after prior local infiltration with $2 \mathrm{ml}$ of $1 \%$ lignocaine.

All injections were given at a rate of $1 \mathrm{ml}$ over $4-5 \mathrm{sec}$ and intrathecal solutions were at room temperature. Thereafter, the patients were placed in the supine position for surgery.

- In group A, spinal anesthesia was performed with $3.5 \mathrm{ml}$ of $0.5 \%$ hyperbaric bupivacaine $(17.5 \mathrm{mg}$ ), plus 5 d d axmedetomidine in $0.5 \mathrm{ml}$ normal saline.

- In group B, $3.5 \mathrm{ml}$ of $0.5 \%$ hyperbaric bupivacaine (17.5mg), plus $25 \mathrm{mg} \mathrm{MgSO} 4$ in $0.5 \mathrm{ml}$ normal saline was injected intrathecally.

- In group C, $3.5 \mathrm{ml}$ of $0.5 \%$ hyperbaric bupivacaine (17.5mg), plus $0.5 \mathrm{ml}$ of normal saline was injected intrathecally.

\section{Data collection:}

The following parameters will be recorded: Vital signs including BP and heart rate every 5 minutes for ${ }^{1 \mathrm{st}} 15$ minutes and every 10 mins until transfer to PACU. Temprature measurement, utilizing thermistor fixed to axilla immediately after spinal anaethesia, then every 15 mins until transfer to PACU. Shivering occurance with Crossley and Mahajan scoring system at the following times: Immediately after spinal anaethesia, every 15 minutes until patient transfere to PACU and every 15 minutes in PACU for two readings.

The method of Crossley and Mahajan was used for measuring the intensity of shivering. The scoring was as follows: $0=$ without shivering; $1=$ piloerection or peripheral vasoconstriction without visible shivering; 2 = muscular activation only in one group of muscles; $3=$ muscular activation in more than one group of muscles without generalized shivering; 4 = generalized shivering.

Twenty-five milligrams of IV meperidine was given when a patient presented grade 3 shivering. The temperature was monitored using a thermistor fixed to axilla before the block, immediately after the block and every $15 \mathrm{~min}$ for $2 \mathrm{~h}$ after the block. Hypothermia and active warming were considered if the core temperature reached $36^{\circ} \mathrm{C}$.

Postoperatively, patients were transferred to the postanesthesia care unit (PACU), monitored and covered with a warm blanket. The PACU temperature was maintained at $25^{\circ} \mathrm{C}$.

\section{Study outcome:}

The primary outcome parameters are the incidence and intensity of postoperative or intraoperative shivering in both groups according to Crossley and Mahajan scoring system. The secondary outcome parameters are the frequency of need and dose of pethidine to control shivering, incidence of sedation, hypothermia and complications including hypotension, bradycardia, nausea and vomiting.

\section{Statistical analysis:}

Data were collected, revised, coded and entered to the Statistical Package for Social Science (IBM SPSS) version 22. The quantitative data were presented as mean, standard deviations and ranges when parametric. Also qualitative variables were presented as number and percentages. The Comparison between groups with qualitative data was done by using Chi-square test. The comparison between more than two groups with quantitative data and parametric distribution were done by using One Way Analysis of Variance (ANOVA). The confidence interval was set to $95 \%$ and the margin of error accepted was set to 5\%. So, the $p$ value was considered significant as the following: $p>0.05$ : Non significant. $p<0.05$ : Significant. $p<0.01$ : Highly significant.

\section{Results}


Table (1): Comparison between the three studied groups regarding demographic data, anthropometric measures.

\begin{tabular}{|c|c|c|c|c|c|c|}
\hline & $\begin{array}{l}\text { Group A } \\
\text { No. }=35\end{array}$ & $\begin{array}{l}\text { Group B } \\
\text { No. }=35\end{array}$ & $\begin{array}{l}\text { Group C } \\
\text { No. }=35\end{array}$ & $\begin{array}{c}\text { Test } \\
\text { value }\end{array}$ & $\begin{array}{c}p- \\
\text { value }\end{array}$ & Sig. \\
\hline $\begin{array}{l}\text { Age: } \\
\quad \text { Mean } \pm \text { SD } \\
\quad \text { Range }\end{array}$ & $\begin{array}{l}31.20 \pm 8.35 \\
18-45\end{array}$ & $\begin{array}{l}33.14 \pm 8.06 \\
18-44\end{array}$ & $\begin{array}{l}31.86 \pm 8.59 \\
18-45\end{array}$ & $0.492 \bullet$ & 0.613 & NS \\
\hline $\begin{array}{l}\text { Sex: } \\
\quad \text { Females } \\
\text { Males }\end{array}$ & $\begin{array}{l}11(31.4 \%) \\
24(68.6 \%)\end{array}$ & $\begin{array}{l}12(34.3 \%) \\
23(65.7 \%)\end{array}$ & $\begin{array}{l}18(51.4 \%) \\
17(48.6 \%)\end{array}$ & $3.441 *$ & 0.179 & NS \\
\hline $\begin{array}{l}\text { Weight: } \\
\quad \text { Mean } \pm \text { SD } \\
\quad \text { Range }\end{array}$ & $\begin{array}{l}82.40 \pm 9.45 \\
60-98\end{array}$ & $\begin{array}{l}84.03 \pm 8.92 \\
59-99\end{array}$ & $\begin{array}{l}78.83 \pm 9.75 \\
59-95\end{array}$ & $2.814 \bullet$ & 0.065 & NS \\
\hline $\begin{array}{l}\text { Height: } \\
\quad \text { Mean } \pm \text { SD } \\
\text { Range }\end{array}$ & $\begin{array}{l}176.86 \pm 11.69 \\
152-196\end{array}$ & $\begin{array}{l}175.50 \pm 11.35 \\
153-194\end{array}$ & $\begin{array}{l}172.54 \pm 10.21 \\
152-190\end{array}$ & $1.384 \bullet$ & 0.255 & NS \\
\hline
\end{tabular}

$p$-value $>0.05$ : Non significant. $p$-value <0.05: Significant. $\quad p$-value <0.01: Highly significant. *: Chi-square test. $\quad \bullet$ One Way ANOVA test.

Table (2): Systolic blood pressure (mmHg).

\begin{tabular}{|c|c|c|c|c|c|c|c|}
\hline SBP & & $\begin{array}{l}\text { Group A } \\
\text { No. }=35\end{array}$ & $\begin{array}{l}\text { Group B } \\
\text { No. }=35\end{array}$ & $\begin{array}{l}\text { Group C } \\
\text { No. }=35\end{array}$ & $\begin{array}{c}\text { Test } \\
\text { value }\end{array}$ & $\begin{array}{c}p- \\
\text { value }\end{array}$ & Sig. \\
\hline Pre-spinal & $\begin{array}{l}\text { Mean } \pm S D \\
\text { Range }\end{array}$ & $\begin{array}{l}126.74 \pm 5.87 \\
110-140\end{array}$ & $\begin{array}{l}128.23 \pm 8.25 \\
118-148\end{array}$ & $\begin{array}{l}129.37 \pm 7.73 \\
110-145\end{array}$ & $1.126^{\bullet}$ & 0.328 & NS \\
\hline After-spinal & $\begin{array}{l}\text { Mean } \pm S D \\
\text { Range }\end{array}$ & $\begin{array}{l}124.43 \pm 5.11 \\
111-135\end{array}$ & $\begin{array}{l}127.94 \pm 7.57 \\
115-140\end{array}$ & $\begin{array}{l}126.94 \pm 6.89 \\
111-140\end{array}$ & $2.631 \bullet$ & 0.077 & NS \\
\hline $5 \mathrm{~min}$ & $\begin{array}{l}\text { Mean } \pm S D \\
\text { Range }\end{array}$ & $\begin{array}{l}120.37 \pm 5.63 \\
105-133\end{array}$ & $\begin{array}{l}120.37 \pm 7.15 \\
103-133\end{array}$ & $\begin{array}{l}121.00 \pm 6.49 \\
103-133\end{array}$ & $0.11 \bullet^{\bullet}$ & 0.895 & NS \\
\hline $10 \mathrm{~m}$ & $\begin{array}{l}\text { Mean } \pm S D \\
\text { Range }\end{array}$ & $\begin{array}{l}116.83 \pm 5.28 \\
100-127\end{array}$ & $\begin{array}{l}115.77 \pm 7.53 \\
100-127\end{array}$ & $\begin{array}{l}116.09 \pm 6.92 \\
100-127\end{array}$ & $0.234 \bullet$ & 0.792 & NS \\
\hline $15 \mathrm{~m}$ & $\begin{array}{l}\text { Mean } \pm \text { SD } \\
\text { Range }\end{array}$ & $\begin{array}{l}113.83 \pm 6.54 \\
90-122\end{array}$ & $\begin{array}{l}113.83 \pm 6.26 \\
100-125\end{array}$ & $\begin{array}{l}112.63 \pm 7.33 \\
90-122\end{array}$ & $0.372 \bullet$ & 0.691 & NS \\
\hline $25 \mathrm{~m}$ & $\begin{array}{l}\text { Mean } \pm S D \\
\text { Range }\end{array}$ & $\begin{array}{l}114.20 \pm 7.40 \\
89-128\end{array}$ & $\begin{array}{l}115.37 \pm 6.92 \\
94-128\end{array}$ & $\begin{array}{l}111.69 \pm 8.60 \\
89-128\end{array}$ & $2.108 \bullet$ & 0.127 & NS \\
\hline $35 \mathrm{~m}$ & $\begin{array}{l}\text { Mean } \pm S D \\
\text { Range }\end{array}$ & $\begin{array}{l}114.80 \pm 8.25 \\
90-129\end{array}$ & $\begin{array}{l}117.54 \pm 7.32 \\
90-130\end{array}$ & $\begin{array}{l}113.20 \pm 8.71 \\
90-129\end{array}$ & $2.564 \bullet$ & 0.082 & NS \\
\hline $45 \mathrm{~m}$ & $\begin{array}{l}\text { Mean } \pm S D \\
\text { Range }\end{array}$ & $\begin{array}{l}116.03 \pm 8.66 \\
95-130\end{array}$ & $\begin{array}{l}119.86 \pm 5.36 \\
100-132\end{array}$ & $\begin{array}{l}115.34 \pm 8.14 \\
95-130\end{array}$ & $3.657 \bullet$ & 0.029 & S \\
\hline $55 \mathrm{~m}$ & $\begin{array}{l}\text { Mean } \pm S D \\
\text { Range }\end{array}$ & $\begin{array}{l}118.09 \pm 8.54 \\
93-132\end{array}$ & $\begin{array}{l}121.57 \pm 5.33 \\
102-136\end{array}$ & $\begin{array}{l}118.29 \pm 8.29 \\
93-132\end{array}$ & $2.364 \bullet$ & 0.099 & NS \\
\hline $65 \mathrm{~m}$ & $\begin{array}{l}\text { Mean } \pm S D \\
\text { Range }\end{array}$ & $\begin{array}{l}119.31 \pm 7.33 \\
98-133\end{array}$ & $\begin{array}{l}122.57 \pm 5.67 \\
100-134\end{array}$ & $\begin{array}{l}119.74 \pm 8.00 \\
98-133\end{array}$ & $2.194 \bullet$ & 0.117 & NS \\
\hline $75 \mathrm{~m}$ & $\begin{array}{l}\text { Mean } \pm S D \\
\text { Range }\end{array}$ & $\begin{array}{l}120.97 \pm 6.67 \\
100-130\end{array}$ & $\begin{array}{l}123.97 \pm 5.59 \\
104-136\end{array}$ & $\begin{array}{l}121.06 \pm 6.96 \\
100-130\end{array}$ & $2.468 \bullet$ & 0.090 & NS \\
\hline $85 \mathrm{~m}$ & $\begin{array}{l}\text { Mean } \pm S D \\
\text { Range }\end{array}$ & $\begin{array}{l}122.94 \pm 6.32 \\
100-132\end{array}$ & $\begin{array}{l}124.51 \pm 5.30 \\
105-137\end{array}$ & $\begin{array}{l}122.26 \pm 6.75 \\
100-132\end{array}$ & $1.236^{\bullet}$ & 0.295 & NS \\
\hline $95 \mathrm{~m}$ & $\begin{array}{l}\text { Mean } \pm S D \\
\text { Range }\end{array}$ & $\begin{array}{l}123.14 \pm 5.65 \\
105-136\end{array}$ & $\begin{array}{l}124.60 \pm 5.56 \\
110-140\end{array}$ & $\begin{array}{l}122.49 \pm 5.99 \\
105-130\end{array}$ & $1.246^{\bullet}$ & 0.292 & NS \\
\hline $105 \mathrm{~m}$ & $\begin{array}{l}\text { Mean } \pm S D \\
\text { Range }\end{array}$ & $\begin{array}{l}124.46 \pm 6.12 \\
104-139\end{array}$ & $\begin{array}{l}126.49 \pm 5.73 \\
115-140\end{array}$ & $\begin{array}{l}124.23 \pm 6.20 \\
104-132\end{array}$ & $1.490 \bullet$ & 0.230 & NS \\
\hline $115 \mathrm{~m}$ & $\begin{array}{l}\text { Mean } \pm S D \\
\text { Range }\end{array}$ & $\begin{array}{l}124.40 \pm 5.94 \\
105-135\end{array}$ & $\begin{array}{l}127.46 \pm 6.17 \\
116-140\end{array}$ & $\begin{array}{l}124.74 \pm 6.45 \\
105-135\end{array}$ & $2.561 \bullet$ & 0.082 & NS \\
\hline
\end{tabular}

$p$-value $>0.05$ : Non significant. $\quad p$-value $<0.05$ : Significant. $\quad p$-value $<0.01$ : Highly significant. $\bullet:$ One Way ANOVA test. 
Table (3): Diastolic blood pressure ( $\mathrm{mmHg}$ ).

\begin{tabular}{|c|c|c|c|c|c|c|}
\hline DBP & $\begin{array}{l}\text { Group A } \\
\text { No. }=35\end{array}$ & $\begin{array}{l}\text { Group B } \\
\text { No. }=35\end{array}$ & $\begin{array}{l}\text { Group C } \\
\text { No. }=35\end{array}$ & $\begin{array}{c}\text { Test } \\
\text { value }\end{array}$ & $\begin{array}{c}p- \\
\text { value }\end{array}$ & Sig. \\
\hline $\begin{array}{l}\text { Pre-spinal: } \\
\quad \text { Mean } \pm \text { SD } \\
\quad \text { Range }\end{array}$ & $\begin{array}{l}84.09 \pm 4.85 \\
70-90\end{array}$ & $\begin{array}{l}84.66 \pm 5.71 \\
70-94\end{array}$ & $\begin{array}{l}84.89 \pm 5.00 \\
70-92\end{array}$ & $0.220 \bullet$ & 0.803 & NS \\
\hline $\begin{array}{l}\text { After-spinal: } \\
\quad \text { Mean } \pm \mathrm{SD} \\
\quad \text { Range }\end{array}$ & $\begin{array}{l}82.09 \pm 5.01 \\
70-90\end{array}$ & $\begin{array}{l}81.91 \pm 5.91 \\
70-90\end{array}$ & $\begin{array}{l}82.60 \pm 5.07 \\
70-90\end{array}$ & $0.156 \bullet$ & 0.856 & NS \\
\hline $\begin{array}{l}\text { 5min: } \\
\quad \text { Mean } \pm \text { SD } \\
\quad \text { Range }\end{array}$ & $\begin{array}{l}77.89 \pm 5.96 \\
65-90\end{array}$ & $\begin{array}{l}76.23 \pm 6.65 \\
58-88\end{array}$ & $\begin{array}{l}77.97 \pm 5.51 \\
58-85\end{array}$ & $0.920 \bullet$ & 0.402 & NS \\
\hline $\begin{array}{l}\text { 10m: } \\
\quad \text { Mean } \pm \text { SD } \\
\quad \text { Range }\end{array}$ & $\begin{array}{l}74.43 \pm 5.29 \\
60-86\end{array}$ & $\begin{array}{l}72.34 \pm 6.20 \\
57-80\end{array}$ & $\begin{array}{l}74.14 \pm 5.99 \\
57-83\end{array}$ & $1.313 \bullet$ & 0.274 & NS \\
\hline $\begin{array}{l}\text { 15m: } \\
\quad \text { Mean } \pm \mathrm{SD} \\
\quad \text { Range }\end{array}$ & $\begin{array}{l}73.26 \pm 5.85 \\
55-84\end{array}$ & $\begin{array}{l}70.74 \pm 5.98 \\
59-82\end{array}$ & $\begin{array}{l}71.00 \pm 6.54 \\
55-80\end{array}$ & $1.783 \bullet$ & 0.173 & NS \\
\hline $\begin{array}{l}\text { 25m: } \\
\quad \text { Mean } \pm \text { SD } \\
\quad \text { Range }\end{array}$ & $\begin{array}{l}72.86 \pm 6.81 \\
50-82\end{array}$ & $\begin{array}{l}71.80 \pm 5.82 \\
60-85\end{array}$ & $\begin{array}{l}70.03 \pm 7.58 \\
50-82\end{array}$ & $1.558 \bullet$ & 0.216 & NS \\
\hline $\begin{array}{l}35 m: \\
\quad \text { Mean } \pm \text { SD } \\
\quad \text { Range }\end{array}$ & $\begin{array}{l}73.97 \pm 6.12 \\
60-82\end{array}$ & $\begin{array}{l}73.80 \pm 5.31 \\
60-82\end{array}$ & $\begin{array}{l}71.57 \pm 6.91 \\
60-82\end{array}$ & $1.659 \bullet$ & 0.195 & NS \\
\hline $\begin{array}{l}\text { 45m: } \\
\quad \text { Mean } \pm \text { SD } \\
\quad \text { Range }\end{array}$ & $\begin{array}{l}75.34 \pm 6.28 \\
60-85\end{array}$ & $\begin{array}{l}75.29 \pm 5.24 \\
63-83\end{array}$ & $\begin{array}{l}73.86 \pm 6.45 \\
62-85\end{array}$ & $0.686 \bullet$ & 0.506 & NS \\
\hline $\begin{array}{l}\text { 55m: } \\
\quad \text { Mean } \pm \text { SD } \\
\quad \text { Range }\end{array}$ & $\begin{array}{l}76.06 \pm 6.67 \\
59-89\end{array}$ & $\begin{array}{l}76.51 \pm 4.97 \\
66-86\end{array}$ & $\begin{array}{l}75.80 \pm 6.52 \\
60-89\end{array}$ & $0.123 \bullet$ & 0.884 & NS \\
\hline $\begin{array}{l}65 m: \\
\quad \text { Mean } \pm \text { SD } \\
\quad \text { Range }\end{array}$ & $\begin{array}{l}76.74 \pm 6.80 \\
60-90\end{array}$ & $\begin{array}{l}77.14 \pm 5.65 \\
60-86\end{array}$ & $\begin{array}{l}76.80 \pm 7.25 \\
60-90\end{array}$ & $0.038 \bullet$ & 0.963 & NS \\
\hline $\begin{array}{l}75 m: \\
\quad \text { Mean } \pm \text { SD } \\
\quad \text { Range }\end{array}$ & $\begin{array}{l}78.63 \pm 6.01 \\
62-88\end{array}$ & $\begin{array}{l}78.74 \pm 5.93 \\
63-88\end{array}$ & $\begin{array}{l}77.69 \pm 6.56 \\
63-88\end{array}$ & $0.309 \bullet$ & 0.735 & NS \\
\hline $\begin{array}{l}\text { 85m: } \\
\quad \text { Mean } \pm \text { SD } \\
\quad \text { Range }\end{array}$ & $\begin{array}{l}80.17 \pm 6.10 \\
60-90\end{array}$ & $\begin{array}{l}78.91 \pm 5.77 \\
65-88\end{array}$ & $\begin{array}{l}79.49 \pm 6.71 \\
60-90\end{array}$ & $0.360 \bullet$ & 0.699 & NS \\
\hline $\begin{array}{l}95 \mathrm{~m}: \\
\quad \text { Mean } \pm \mathrm{SD} \\
\quad \text { Range }\end{array}$ & $\begin{array}{l}81.60 \pm 5.11 \\
69-90\end{array}$ & $\begin{array}{l}79.43 \pm 5.92 \\
66-90\end{array}$ & $\begin{array}{l}80.57 \pm 5.86 \\
69-89\end{array}$ & $1.297 \bullet$ & 0.278 & NS \\
\hline $\begin{array}{l}\text { 105m: } \\
\quad \text { Mean } \pm \text { SD } \\
\quad \text { Range }\end{array}$ & $\begin{array}{l}82.11 \pm 5.05 \\
70-90\end{array}$ & $\begin{array}{l}80.83 \pm 6.09 \\
65-91\end{array}$ & $\begin{array}{l}81.71 \pm 4.82 \\
70-90\end{array}$ & $0.530 \bullet$ & 0.590 & NS \\
\hline $\begin{array}{l}115 \mathrm{~m}: \\
\quad \text { Mean } \pm \mathrm{SD} \\
\quad \text { Range }\end{array}$ & $\begin{array}{l}82.11 \pm 5.49 \\
68-90\end{array}$ & $\begin{array}{l}81.83 \pm 6.12 \\
64-90\end{array}$ & $\begin{array}{l}82.00 \pm 5.53 \\
68-90\end{array}$ & $0.022 \bullet$ & 0.978 & NS \\
\hline
\end{tabular}

$p$-value $>0.05$ : Non significant.

$p$-value $<0.05$ : Significant.

$p$-value $<0.01$ : Highly significant.

$\bullet$ One Way ANOVA test. 
Table (4): Heart rate.

\begin{tabular}{|c|c|c|c|c|c|c|c|}
\hline HR & & $\begin{array}{l}\text { Group A } \\
\text { No. }=35\end{array}$ & $\begin{array}{l}\text { Group B } \\
\text { No. }=35\end{array}$ & $\begin{array}{l}\text { Group C } \\
\text { No. }=35\end{array}$ & $\begin{array}{c}\text { Test } \\
\text { value }\end{array}$ & $\begin{array}{c}p- \\
\text { value }\end{array}$ & Sig. \\
\hline Pre-spinal & $\begin{array}{l}\text { Mean } \pm \text { SD } \\
\text { Range }\end{array}$ & $\begin{array}{l}78.77 \pm 11.34 \\
55-99\end{array}$ & $\begin{array}{l}79.57 \pm 11.73 \\
54-99\end{array}$ & $\begin{array}{l}81.74 \pm 10.54 \\
59-97\end{array}$ & $0.658 \bullet$ & 0.520 & NS \\
\hline After-spinal & $\begin{array}{l}\text { Mean } \pm \text { SD } \\
\text { Range }\end{array}$ & $\begin{array}{l}79.46 \pm 10.53 \\
57-96\end{array}$ & $\begin{array}{l}79.14 \pm 10.82 \\
53-98\end{array}$ & $\begin{array}{l}82.31 \pm 10.19 \\
58-98\end{array}$ & $0.966^{\bullet}$ & 0.384 & NS \\
\hline $5 \mathrm{~min}$ & $\begin{array}{l}\text { Mean } \pm \text { SD } \\
\text { Range }\end{array}$ & $\begin{array}{l}77.86 \pm 9.76 \\
58-94\end{array}$ & $\begin{array}{l}77.57 \pm 9.95 \\
54-96\end{array}$ & $\begin{array}{l}80.09 \pm 9.63 \\
59-96\end{array}$ & $0.693 \bullet$ & 0.502 & NS \\
\hline $10 \mathrm{~m}$ & $\begin{array}{l}\text { Mean } \pm \text { SD } \\
\text { Range }\end{array}$ & $\begin{array}{l}75.94 \pm 10.13 \\
51-98\end{array}$ & $\begin{array}{l}76.11 \pm 9.86 \\
57-98\end{array}$ & $\begin{array}{l}78.57 \pm 8.89 \\
57-92\end{array}$ & $0.814 \bullet$ & 0.446 & NS \\
\hline $15 \mathrm{~m}$ & $\begin{array}{l}\text { Mean } \pm \text { SD } \\
\text { Range }\end{array}$ & $\begin{array}{l}74.74 \pm 9.58 \\
54-93\end{array}$ & $\begin{array}{l}74.57 \pm 9.19 \\
55-93\end{array}$ & $\begin{array}{l}77.03 \pm 9.35 \\
55-93\end{array}$ & $0.749 \bullet$ & 0.475 & NS \\
\hline $25 \mathrm{~m}$ & $\begin{array}{l}\text { Mean } \pm \text { SD } \\
\text { Range }\end{array}$ & $\begin{array}{l}74.03 \pm 8.55 \\
56-90\end{array}$ & $\begin{array}{l}73.74 \pm 8.70 \\
57-90\end{array}$ & $\begin{array}{l}76.83 \pm 8.27 \\
59-90\end{array}$ & $1.406^{\bullet}$ & 0.250 & NS \\
\hline $35 \mathrm{~m}$ & $\begin{array}{l}\text { Mean } \pm \text { SD } \\
\text { Range }\end{array}$ & $\begin{array}{l}74.63 \pm 9.18 \\
58-92\end{array}$ & $\begin{array}{l}73.97 \pm 10.63 \\
51-94\end{array}$ & $\begin{array}{l}76.86 \pm 9.47 \\
60-94\end{array}$ & $0.837 \bullet$ & 0.436 & NS \\
\hline $45 \mathrm{~m}$ & $\begin{array}{l}\text { Mean } \pm \text { SD } \\
\text { Range }\end{array}$ & $\begin{array}{l}74.23 \pm 8.69 \\
57-90\end{array}$ & $\begin{array}{l}74.71 \pm 10.08 \\
55-98\end{array}$ & $\begin{array}{l}76.74 \pm 9.63 \\
62-98\end{array}$ & $0.692 \bullet$ & 0.503 & NS \\
\hline $55 \mathrm{~m}$ & $\begin{array}{l}\text { Mean } \pm \text { SD } \\
\text { Range }\end{array}$ & $\begin{array}{l}74.17 \pm 9.00 \\
57-90\end{array}$ & $\begin{array}{l}74.34 \pm 9.94 \\
57-95\end{array}$ & $\begin{array}{l}75.97 \pm 9.36 \\
57-95\end{array}$ & $0.388 \bullet$ & 0.680 & NS \\
\hline $65 \mathrm{~m}$ & $\begin{array}{l}\text { Mean } \pm \text { SD } \\
\text { Range }\end{array}$ & $\begin{array}{l}73.89 \pm 9.68 \\
54-94\end{array}$ & $\begin{array}{l}75.43 \pm 10.51 \\
54-94\end{array}$ & $\begin{array}{l}76.00 \pm 9.60 \\
54-94\end{array}$ & $0.424 \bullet$ & 0.656 & NS \\
\hline $75 \mathrm{~m}$ & $\begin{array}{l}\text { Mean } \pm \text { SD } \\
\text { Range }\end{array}$ & $\begin{array}{l}74.69 \pm 9.04 \\
58-92\end{array}$ & $\begin{array}{l}76.20 \pm 10.22 \\
53-95\end{array}$ & $\begin{array}{l}76.80 \pm 8.90 \\
58-95\end{array}$ & $0.470 \bullet$ & 0.626 & NS \\
\hline $85 \mathrm{~m}$ & $\begin{array}{l}\text { Mean } \pm \text { SD } \\
\text { Range }\end{array}$ & $\begin{array}{l}75.17 \pm 8.99 \\
57-90\end{array}$ & $\begin{array}{l}76.09 \pm 9.74 \\
51-92\end{array}$ & $\begin{array}{l}77.14 \pm 9.61 \\
59-92\end{array}$ & $0.381 \bullet$ & 0.684 & NS \\
\hline $95 \mathrm{~m}$ & $\begin{array}{l}\text { Mean } \pm \text { SD } \\
\text { Range }\end{array}$ & $\begin{array}{l}76.00 \pm 8.63 \\
58-92\end{array}$ & $\begin{array}{l}77.14 \pm 9.42 \\
59-95\end{array}$ & $\begin{array}{l}78.00 \pm 9.63 \\
60-95\end{array}$ & $0.413 \bullet$ & 0.663 & NS \\
\hline $105 \mathrm{~m}$ & $\begin{array}{l}\text { Mean } \pm \text { SD } \\
\text { Range }\end{array}$ & $\begin{array}{l}76.94 \pm 8.98 \\
59-94\end{array}$ & $\begin{array}{l}77.89 \pm 8.93 \\
60-92\end{array}$ & $\begin{array}{l}78.37 \pm 9.11 \\
62-94\end{array}$ & $0.228 \bullet$ & 0.797 & NS \\
\hline $115 \mathrm{~m}$ & $\begin{array}{l}\text { Mean } \pm \text { SD } \\
\text { Range }\end{array}$ & $\begin{array}{l}77.49 \pm 9.53 \\
57-93\end{array}$ & $\begin{array}{l}78.46 \pm 9.14 \\
57-94\end{array}$ & $\begin{array}{l}79.09 \pm 9.37 \\
61-94\end{array}$ & $0.260 \bullet$ & 0.771 & \\
\hline
\end{tabular}

$p$-value $>0.05$ : Non significant. $\quad p$-value $<0.05$ : Significant. $\quad p$-value $<0.01$ : Highly significant. $\bullet$ : One Way ANOVA test.

Table (5): Temperature.

\begin{tabular}{|c|c|c|c|c|c|c|c|}
\hline Temperature & & $\begin{array}{l}\text { Group A } \\
\text { No. }=35\end{array}$ & $\begin{array}{l}\text { Group B } \\
\text { No. }=35\end{array}$ & $\begin{array}{l}\text { Group C } \\
\text { No. }=35\end{array}$ & $\begin{array}{c}\text { Test } \\
\text { value }\end{array}$ & $\begin{array}{c}p- \\
\text { value }\end{array}$ & Sig. \\
\hline After-spinal & $\begin{array}{l}\text { Mean } \pm \text { SD } \\
\text { Range }\end{array}$ & $\begin{array}{l}37.13 \pm 0.08 \\
36.99-37.2\end{array}$ & $\begin{array}{l}37.16 \pm 0.06 \\
37-37.2\end{array}$ & $\begin{array}{l}37.11 \pm 0.08 \\
36.99-37.2\end{array}$ & $4.915 \bullet$ & 0.009 & HS \\
\hline $15 \mathrm{~m}$ & $\begin{array}{l}\text { Mean } \pm \text { SD } \\
\text { Range }\end{array}$ & $\begin{array}{l}37.12 \pm 0.07 \\
36.95-37.2\end{array}$ & $\begin{array}{l}37.12 \pm 0.08 \\
36.8-37.2\end{array}$ & $\begin{array}{l}37.08 \pm 0.09 \\
36.8-37.19\end{array}$ & $2.942 \bullet$ & 0.057 & NS \\
\hline $30 \mathrm{~m}$ & $\begin{array}{l}\text { Mean } \pm \text { SD } \\
\text { Range }\end{array}$ & $\begin{array}{l}37.08 \pm 0.08 \\
36.92-37.2\end{array}$ & $\begin{array}{l}37.05 \pm 0.11 \\
36.8-37.19\end{array}$ & $\begin{array}{l}37.00 \pm 0.11 \\
36.8-37.2\end{array}$ & $5.146^{\bullet}$ & 0.007 & HS \\
\hline $45 \mathrm{~m}$ & $\begin{array}{l}\text { Mean } \pm \text { SD } \\
\text { Range }\end{array}$ & $\begin{array}{l}37.05 \pm 0.09 \\
36.86-37.18\end{array}$ & $\begin{array}{l}36.99 \pm 0.17 \\
36.55-37.18\end{array}$ & $\begin{array}{l}36.94 \pm 0.14 \\
36.69-37.16\end{array}$ & $5.789 \bullet$ & 0.004 & HS \\
\hline $60 \mathrm{~m}$ & $\begin{array}{l}\text { Mean } \pm \text { SD } \\
\text { Range }\end{array}$ & $\begin{array}{l}36.99 \pm 0.10 \\
36.84-37.19\end{array}$ & $\begin{array}{l}36.96 \pm 0.20 \\
36.5-37.19\end{array}$ & $\begin{array}{l}36.89 \pm 0.16 \\
36.5-37.15\end{array}$ & $3.657 \bullet$ & 0.029 & HS \\
\hline $75 \mathrm{~m}$ & $\begin{array}{l}\text { Mean } \pm \text { SD } \\
\text { Range }\end{array}$ & $\begin{array}{l}36.94 \pm 0.11 \\
36.82-37.19\end{array}$ & $\begin{array}{l}36.85 \pm 0.22 \\
36.4-37.19\end{array}$ & $\begin{array}{l}36.83 \pm 0.19 \\
36.5-37.16\end{array}$ & $3.732 \bullet$ & 0.027 & HS \\
\hline $90 \mathrm{~m}$ & $\begin{array}{l}\text { Mean } \pm \text { SD } \\
\text { Range }\end{array}$ & $\begin{array}{l}36.85 \pm 0.12 \\
36.7-37.10\end{array}$ & $\begin{array}{l}36.52 \pm 0.25 \\
36.31-37.17\end{array}$ & $\begin{array}{l}36.55 \pm 0.22 \\
36.32-37.17\end{array}$ & $27.905 \bullet$ & 0.000 & HS \\
\hline $105 \mathrm{~m}$ & $\begin{array}{l}\text { Mean } \pm \text { SD } \\
\text { Range }\end{array}$ & $\begin{array}{l}36.73 \pm 0.12 \\
36.5-37.19\end{array}$ & $\begin{array}{l}36.50 \pm 0.27 \\
36.3-37.19\end{array}$ & $\begin{array}{l}36.51 \pm 0.27 \\
36.1-37.17\end{array}$ & $11.077 \bullet$ & 0.000 & HS \\
\hline $120 \mathrm{~m}$ & $\begin{array}{l}\text { Mean } \pm \text { SD } \\
\text { Range }\end{array}$ & $\begin{array}{l}36.68 \pm 0.12 \\
36.4-37.15\end{array}$ & $\begin{array}{l}36.45 \pm 0.29 \\
36.3-37.17\end{array}$ & $\begin{array}{l}36.47 \pm 0.31 \\
36.1-37.2\end{array}$ & $8.759 \bullet$ & 0.000 & HS \\
\hline
\end{tabular}

$p$-value $>0.05$ : Non significant. $\quad p$-value $<0.05$ : Significant. $\quad p$-value $<0.01$ : Highly significant. $\bullet:$ One Way ANOVA test. 
Table (6): Comparison between the three studied groups regarding incidence of shivering.

\begin{tabular}{lcccccc}
\hline $\begin{array}{l}\text { Incidence of } \\
\text { shivering }\end{array}$ & $\begin{array}{c}\text { Group A } \\
\text { No. }=35\end{array}$ & $\begin{array}{c}\text { Group B } \\
\text { No. }=35\end{array}$ & $\begin{array}{c}\text { Group C } \\
\text { No. }=35\end{array}$ & $\begin{array}{c}\text { Test } \\
\text { value }\end{array}$ & $\begin{array}{c}p \text { - } \\
\text { value }\end{array}$ & $\begin{array}{c}\text { Sig. } \\
\text { No (G0) }\end{array}$ \\
\hline Yes (G1-4) & $9(74.3 \%)$ & $23(65.7 \%)$ & $15(42.9 \%)$ & $7.763^{*}$ & 0.020 & $\mathrm{~S}$ \\
\hline
\end{tabular}

$p$-value $>0.05$ : Non significant. $p$-value <0.05: Significant. $p$-value <0.01: Highly significant. *: Chi-square test.

Table (7): Comparison between the two drugs regarding incidence of shivering.

\begin{tabular}{lccccc}
\hline $\begin{array}{l}\text { Incidence of } \\
\text { shivering }\end{array}$ & $\begin{array}{c}\text { Group A } \\
\text { No. }=35\end{array}$ & $\begin{array}{c}\text { Group B } \\
\text { No. }=35\end{array}$ & $\begin{array}{c}\text { Test } \\
\text { value }\end{array}$ & $\begin{array}{c}p \text { - } \\
\text { value }\end{array}$ & Sig. \\
\hline No (G0) & $26(74.3 \%)$ & $23(65.7 \%)$ & 0.612 & 0.434 \\
Yes (G1-4) & $9(25.7 \%)$ & $12(34.3 \%)$ & & & NS \\
\hline
\end{tabular}

Table (8): Comparison between the three studied groups regarding shivering grade.

\begin{tabular}{|c|c|c|c|c|c|c|}
\hline Shivering & $\begin{array}{l}\text { Group A } \\
\text { No. }=35\end{array}$ & $\begin{array}{l}\text { Group B } \\
\text { No. }=35\end{array}$ & $\begin{array}{l}\text { Group C } \\
\text { No. }=35\end{array}$ & $\begin{array}{c}\text { Test } \\
\text { value }\end{array}$ & $\begin{array}{c}p- \\
\text { value }\end{array}$ & Sig. \\
\hline Grade 0 & $26(74.3 \%)$ & $23(65.7 \%)$ & $15(42.9 \%)$ & 7.763 & 0.020 & S \\
\hline Grade I & $0(0.0 \%)$ & $0(0.0 \%)$ & $0(0.0 \%)$ & & & - \\
\hline Grade II & $4(11.4 \%)$ & $6(17.1 \%)$ & $2(5.7 \%)$ & 2.258 & 0.323 & NS \\
\hline Grade III & $2(5.7 \%)$ & $2(5.7 \%)$ & $3(8.6 \%)$ & 0.306 & 0.858 & NS \\
\hline Grade IV & $3(8.6 \%)$ & $4(11.4 \%)$ & $15(42.9 \%)$ & 15.296 & 0.001 & HS \\
\hline
\end{tabular}

$p$-value $>0.05$ : Non significant. $\quad p$-value $<0.05$ : Significant. $p$-value $<0.01$ : Highly significant. $*$ : Chi-square test.

Table (8): Comparison between the two drugs regarding shivering grade.

\begin{tabular}{|c|c|c|c|c|c|}
\hline Shivering & $\begin{array}{l}\text { Group A } \\
\text { No. }=35\end{array}$ & $\begin{array}{l}\text { Group B } \\
\text { No. }=35\end{array}$ & $\begin{array}{c}\text { Test } \\
\text { value }\end{array}$ & $\begin{array}{c}p- \\
\text { value }\end{array}$ & Sig. \\
\hline \multicolumn{6}{|c|}{ Incidence of shivering: } \\
\hline Grade 0 & $26(74.3 \%)$ & $23(65.7 \%)$ & 0.612 & 0.434 & NS \\
\hline Grade I & $0(0.0 \%)$ & $0(0.0 \%)$ & & & \\
\hline Grade II & $4(11.4 \%)$ & $6(17.1 \%)$ & 0.467 & 0.494 & NS \\
\hline Grade III & $2(5.7 \%)$ & $2(5.7 \%)$ & 0.000 & 1.000 & NS \\
\hline Grade IV & $3(8.6 \%)$ & $4(11.4 \%)$ & 0.159 & 0.690 & NS \\
\hline
\end{tabular}

$p$-value $>0.05$ : Non significant. $\quad p$-value <0.05: Significant. $p$-value <0.01: Highly significant. $*$ : Chi-square test.

Table (9): Comparison between the three studied groups regarding need to pethidine.

\begin{tabular}{|c|c|c|c|c|c|c|}
\hline $\begin{array}{l}\text { Recurrence of } \\
\text { shivering after } 1 \text { st dose } \\
\text { of pethidine }\end{array}$ & $\begin{array}{l}\text { Group A } \\
\text { No. }=35\end{array}$ & $\begin{array}{l}\text { Group B } \\
\text { No. }=35\end{array}$ & $\begin{array}{l}\text { Group C } \\
\text { No. }=35\end{array}$ & $\begin{array}{c}\text { Test } \\
\text { value }\end{array}$ & $\begin{array}{c}p- \\
\text { value }\end{array}$ & Sig. \\
\hline \multicolumn{7}{|l|}{$\begin{array}{l}\text { Need of } 1 \text { st dose } \\
\text { of pethidine: }\end{array}$} \\
\hline $\begin{array}{l}\text { No } \\
\text { Yes }\end{array}$ & $\begin{array}{l}26(74.3 \%) \\
9(25.7 \%)\end{array}$ & $\begin{array}{l}23(65.7 \%) \\
12(34.3 \%)\end{array}$ & $\begin{array}{l}14(40.0 \%) \\
21(60.0 \%)\end{array}$ & $9.286^{*}$ & 0.010 & $S$ \\
\hline \multicolumn{7}{|l|}{$\begin{array}{l}\text { Need of } 2 \text { nd dose } \\
\text { of pethindine: }\end{array}$} \\
\hline
\end{tabular}

$p$-value $>0.05$ : Non significant. $\quad p$-value $<0.05$ : Significant. $p$-value $<0.01$ : Highly significant. $*$ : Chi-square test. 
Table (10): Comparison between the two drugs regarding need to pethidine.

\begin{tabular}{|c|c|c|c|c|c|}
\hline $\begin{array}{l}\text { Recurrence of } \\
\text { shivering after } 1 \text { st dose } \\
\text { of pethidine }\end{array}$ & $\begin{array}{l}\text { Group A } \\
\text { No. }=35\end{array}$ & $\begin{array}{l}\text { Group B } \\
\text { No. }=35\end{array}$ & $\begin{array}{c}\text { Test } \\
\text { value }\end{array}$ & $\begin{array}{c}p- \\
\text { value }\end{array}$ & Sig. \\
\hline \multicolumn{6}{|l|}{$\begin{array}{l}\text { Need of } 1 \text { st dose } \\
\text { of pethidine: }\end{array}$} \\
\hline $\begin{array}{l}\text { No } \\
\text { Yes }\end{array}$ & $\begin{array}{l}26(74.3 \%) \\
9(25.7 \%)\end{array}$ & $\begin{array}{l}23(65.7 \%) \\
12(34.3 \%)\end{array}$ & 0.612 & 0.434 & NS \\
\hline \multicolumn{6}{|l|}{$\begin{array}{l}\text { Need of } 2 \text { nd dose } \\
\text { of pethindine: }\end{array}$} \\
\hline $\begin{array}{l}\text { No } \\
\text { Yes }\end{array}$ & $\begin{array}{l}32(91.4 \%) \\
3(8.6 \%)\end{array}$ & $\begin{array}{l}32(91.4 \%) \\
3(8.6 \%)\end{array}$ & 0.000 & 1.000 & NS \\
\hline
\end{tabular}

$p$-value $>0.05$ : Non significant. $\quad p$-value <0.05: Significant. $p$-value <0.01: Highly significant. *: Chi-square test. $\bullet:$ One way ANOVA test.

\section{Discussion}

Spinal anaesthesia (SA) is a very reliable and convenient technique, especially for procedures in which patient consciousness must be maintained to detect intraoperative complications. However, shivering is a common complication after SA, with an incidence of $40-60 \%$ in patients who undergo SA, especially when large amounts of cold intraluminal irrigating fluids are used. SA impairs thermoregulation, inhibits tonic vasoconstriction, and causes the redistribution of core heat from the trunk to the peripheral tissue [9].

Various causes such as pain, decreased sympathetic activity, uncontrolled spinal reflux, release of pyrogen, cytokines following surgery, or adrenal suppression, etc. have been suggested as causative factors of shivering, however, thermo-regulation disorders caused by hypothermia $\left(0.5-1{ }^{\circ} \mathrm{C}\right.$ decrease in central heating) has been accepted as the most common factor [10].

Shivering interferes with proper monitoring and is associated with several adverse effects, as it increases the circulating catecholamine, heart rate, cardiac output, minute ventilation, patient oxygen consumption, metabolic $\mathrm{CO} 2$ production, lactic acid level, intraocular and intracranial pressure, and postoperative pain from surgical incision stretching [11].

In this study we compared the efficacy of intrathecal dexmedetomidine versus intrathecal magnesium sulphate for reducing the severity and incidence of post-spinal shivering in lower limb orthopedic surgeries.

Our results showed that all patients in the three groups were comparable regarding demographic data (including age, sex, weight and height).
Our results showed that all patients in the three groups were comparable regarding intraoperative hemodynamics (SBP, DBP and HR). Omar et al. [12] compared the effects of intrathecal dexmedetomidine versus intrathecal magnesium sulfate on the prevention of post spinal anesthesia shivering and found that all the patients were comparable regarding their SBP, DBP and HR throughout the study.

Regarding temperature, though difference in decreased core temperature was clinically insignificant, there was statistical significant difference between the three groups at almost all times as temperature at dexmedetomidine group was higher than magnesium group but the temperature at controlled group was lower than other two groups. Faiz et al. [13] demonstrated that core temperature was reduced in the $\mathrm{MgSO} 4$ group 30min after block administration. Indeed, injection of $\mathrm{MgSO} 4$ is known to result in reduction of core temperature following anesthesia. Omar et al. [12] found that the tympanic temperature remained above $36^{\circ} \mathrm{C}$ in all the patients (who recived intrathecal dexmedetomidine and intrathecal magnesium sulfate and the controled group) at all times, and no patient required active warming.

Regarding shivering, we found that $\mathrm{C}$ group showed statistically significant higher number of total patients who developed shivering than B group and A group. 9 patients (25.7\%) in dexmedetomidine group (group A), 12 patients (34.3\%) in magnesium sulfate group (group B), and 20 patients $(57.1 \%)$ in control group (group C).

In sixty patients who underwent lower abdominal surgery, Abdel Hamid and El-Lakany [14] concluded that adding 5 oxmedetomidine to intrathecal bupivacaine improved the characteristics 
of the spinal block, with less postoperative analgesic requirements and a lower incidence of shivering than the placebo with no sedation or other complications. Shivering occurred in $6.5 \%$ patients in the group I (dexmedetomidine), and in $41.9 \%$ patients in group II (placebo).

Ellakany et al. [7] administered the same intrathecal dexmedetomidine dose $(5 \mu \mathrm{g})$ and found that shivering developed in $(20 \%)$ in group D (dexmedetomidine) and (92\%) in group S (placebo). So concluded that intrathecal dexmedetomidine effectively lowered the incidence of shivering following SA in patients who underwent lower abdominal surgery. Moawad and Elawdy [5] studied the effect of adding dexmedetomidine to intrathecal bupivacaine at a higher dose $(10 \mathrm{gg})$ than that in our study $(5 \mu \mathrm{g})$. They found that it significantly decreased the incidence and degree of shivering in patients undergoing TURP. The incidence of shivering was significantly reduced in group $\mathrm{D}$ (dexmedetomidine) $(15 \%)$ compared with $(57 \%)$ in group C (control).

Faiz et al. [13] concluded that an intrathecal injection of $\mathrm{MgSO}_{4}(25 \mathrm{mg})$ improved perioperative shivering in female patients who underwent an elective caesarean section.

Kawakami et al. [15] evaluated the effect of perioperative magnesium on prevention of shivering. They showed a significantly lower incidence of shivering in patients who received magnesium with IV administration, epidural administration, and intrathecal administration, but not with intraarticular administration. Omar et al. [12] found that control group (C) had a significantly higher number of patients $(60 \%)$ who developed shivering than magnesium groups (M) (22.9\%) and dexmedetomidine group (D) (14.3\%).

In our study, $\mathrm{C}$ group showed statistically significant higher number of patients who developed grade IV shivering than B group and A group. Omar et al. [12] found that control group (C) had a significantly higher number of patients who developed grade IV shivering than Magnesium group (M) and Dexmedetomidine group (D).

In our study, there was significant difference between the three studied groups in number of patients who needed pethidine to treat shivering, as 9 patients $(25.7 \%)$ required pethidine in group A which was statistically significant when compared to group B in which 12 patients (34.3\%) required pethidine while group $\mathrm{C} 21$ patients needed pethidine which was highly significant in comparison with the other two groups.
Omar et al. [12] found that control group (C) had a significantly higher number of patients who needed meperidine to treat shivering than groups Magnesium and Dexmedetomidine. They concluded that intrathecal injections of both dexmedetomidine and magnesium sulfate were effective in reducing the incidence of post-spinal anaesthesia shivering and this goes with our study.

Also the need for a second dose of pethidine in control group (C) was highly significant in comparison with the other two groups, and was non-significant among group A (dexmedetomidine) and group B (magnesium sulphate).

\section{Conclusion:}

Our study viewed that intrathecal administration of dexmedetomidine or magnesium sulphate as adjuvant to heavy bupivacaine in spinal anesthesia, reduced incidence and severity of shivering in patients presented for lower limb orthopedic surgery with non-significant difference among both adjuvants.

\section{References}

1- ABDEL-WAHAB A.H., OSMAN E.A. and AHMED A.Y. Comparison of postoperative analgesic effects of two doses of dexamethasone in ultrasound-guided transversus abdominis plane block for inguinal hernia repair: A randomized controlled trial. Ain-Shams Journal of Anesthesiology, 13 (1): 1-8, 2021.

2- LOPEZ M.B.: Postanaesthetic shivering - from pathophysiology to prevention. Rom J. Anaesth Intensive Care, 25: 73-81, 2018.

3- JAIN A., GRAY M., SLISZ S., HAYMORE J., BADJATIA N. and KULSTAD E.: Shivering Treatments for Targeted Temperature Management: A Review. J. Neurosci Nurs., 50: 63-7, 2018.

4- SHARMA S., RAGHU K., NIKHIL N., RAJARAM G., KUMAR S. and SINGH S.: Prophylactic administration of ondansetron for prevention of shivering during spinal anesthesia. Indian Anaesth Forum, 19: 11, 2018.

5- MOAWAD H.E.S. and ELAWDY M.M.: Efficacy of intrathecal dexmedetomidine in prevention of shivering in patients undergoing transurethral prostatectomy: A randomized controlled trial. Egypt J. Anaesth., 31 (2): 178-81, 2015.

6- LAMONTAGNE C., LESAGE S., VILLENEUVE E. LIDZBORSKI E., DERSTENFELD A. and CROCHETIÈRE C.: Intravenous dexmedetomidine for the treatment of shivering during cesarean delivery under neuraxial anesthesia: A randomizedcontrolled trial. Can J. Anesth/J. Can Anesth., 66: 762-11, 2019.

7- ELLAKANY M., ABDELHAMID S.A. and GIRGIS M.: Intrathecal dexmedetomidine or meperidine for post spinal shivering. Int. J. Anesth. Anesth., 1 (2): 1-4, 2014.

8- ANUPAMA W., PAPIYA S. and JALEEL D.: Magnesium sulfate only slightly reduces the shivering threshold in 
humans. British Journal of Anesthesia, 94 (6): 756-762, 2005.

9- MOEEN S.M. and MOEEN A.M.: Intrathecal dexamethasone vs. meperidine for prevention of shivering during transurethral prostatectomy: A randomized controlled trial. Acta. Anaethesiol. Scand, 61: 749-57, 2017.

10- RONALD D.M.: Textbook of Anesthesia. USA: Churchill living ton, 2005.

11-De WITTE J. and SESSLER D.I.: Perioperative shivering: physiology and pharmacology. Anesthesiology, 96: 46784, 2002.

12- OMAR H., ABOELLA W.A., HASSAN M.M., HASSAN A., HASSAN P., ELSHALL A., KHALED D., MOSTAFA M., TAWADROS P.Z., ELDIN M.H., WEDAD M. and ABDELHAMID B.M.: Comparative study between intrathecal dexmedetomidine and intrathecal magnesium sulfate for the prevention of post-spinal anaesthesia shivering in uroscopic surgery. BMC Anesthesiology, 19: 190, 2019.

13-FAIZ S.H.R., RAHIMZADEH P., IMANI F. and BAKHTIARI A.: Intrathecal injection of magnesium sulfate: Shivering prevention during cesarean section: A randomized, double-blinded, controlled study. Korean Journal of Anesthesiology, 65 (4): 293, 2013.

14-ABDELHAMID S.A. and EL-LAKANY M.H.: Intrathecal dexmedetomidine: useful or not? J. Anesth. Clin. Res., 4: 351, 2013.

15- KAWAKAMI H., NAKAJIMA D., MIHARA T., SATO H., GOTO T.: Effectiveness of magnesium in preventing shivering in surgical patients: A systematic review and meta-analysis. Anesthesia \& Analgesia, 129 (3): 689-700, 2019. 


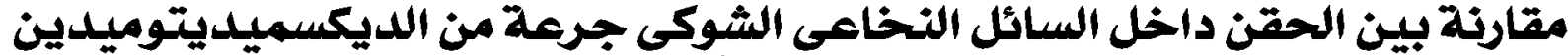

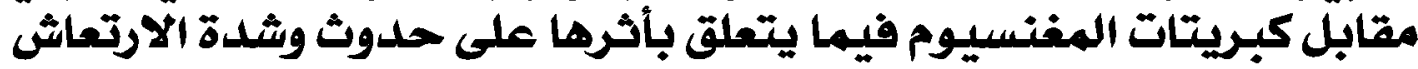

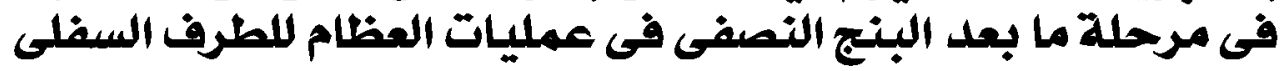

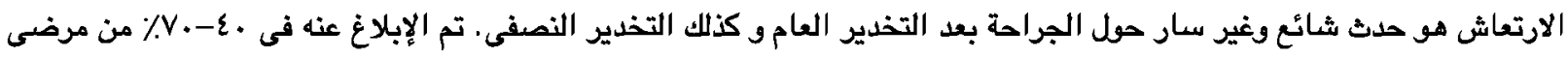

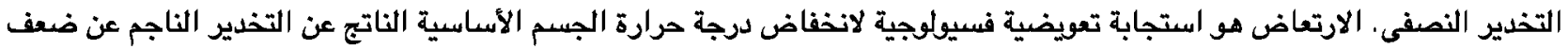

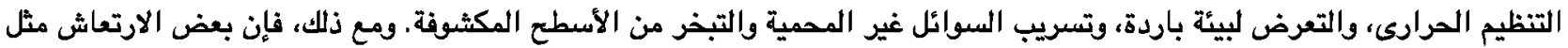
الهزات لا يكون منظماً للحرارة.

يظهر الارتعاش بعد التخدير النصفى بدرجات مختلفة تتراوح من الانتصاب الشعرى فى الشكل الخفيف إلى الانقباض العام اللعضلات الهيكلية فى الشكل الحاد الذى يتداخل مع معدل النبض وضغط الدم النم ومراقبة تخطيط القلب الكهربائى.

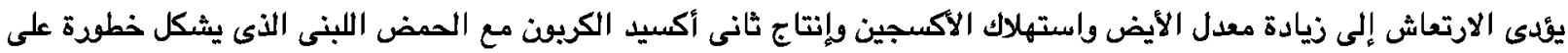

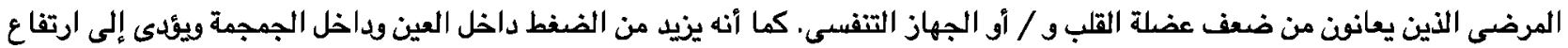

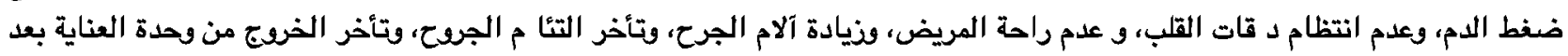

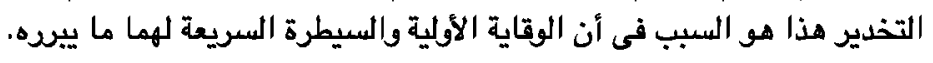

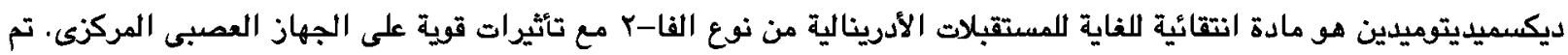

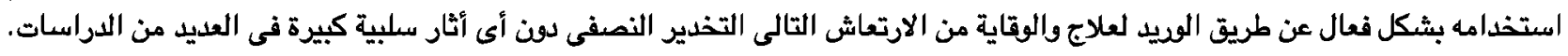

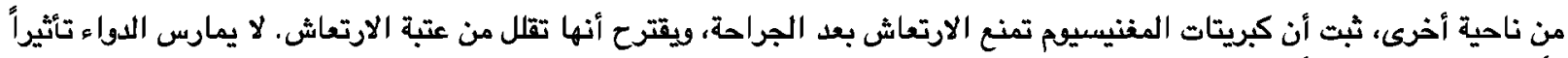

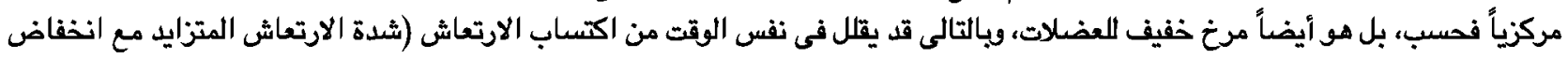
درجة حرارة الجسم المتقدمة).

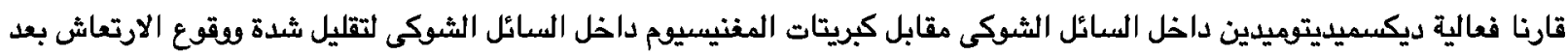

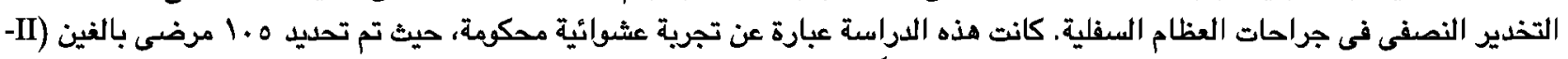

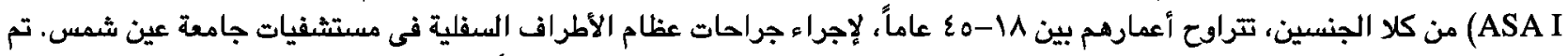

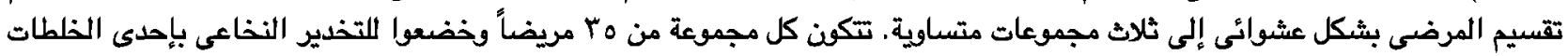

التالية:

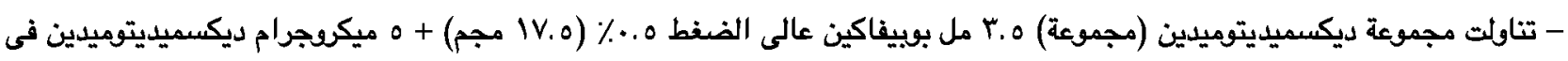

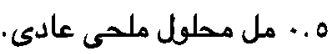

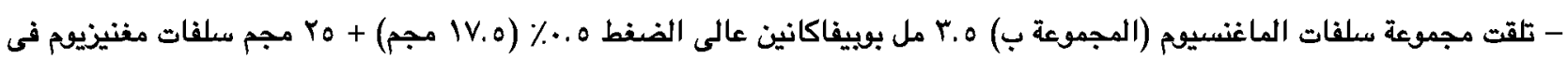

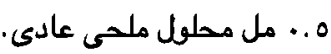

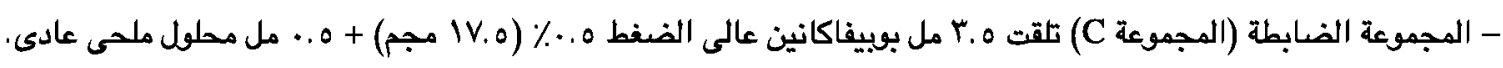

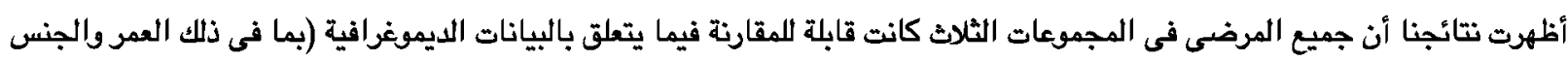

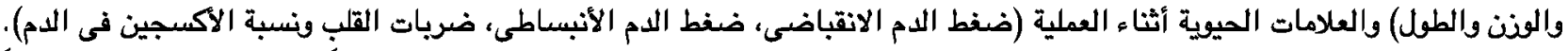

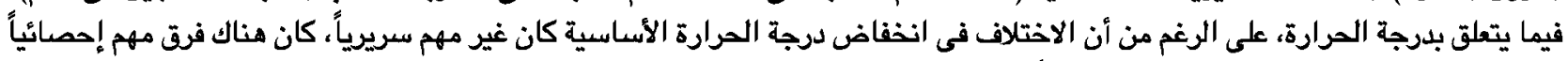
بين المجموعات الثلاث في جميع الأواقات تقريباً.

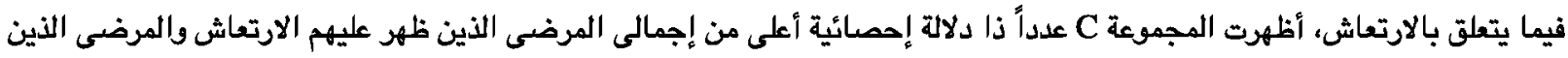

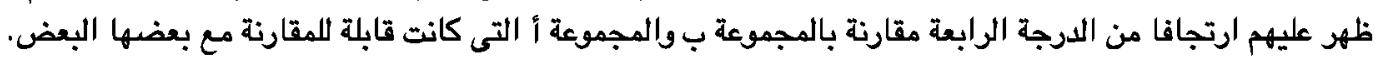

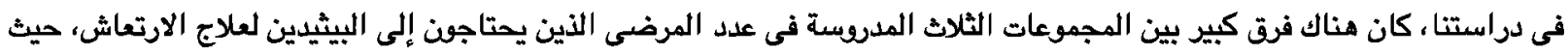

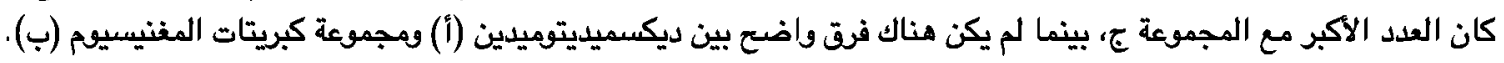

